\title{
0222-P1020 Insulin resistance in patients with hypothyroidism or hyperthyroidism without hepatosteatosis
}

SO Keskek, S Kirim, M Tasdemir, D Tuzun, G Ortoglu, T Saler

Numune Education and Training Hospital, Department of Internal Medicine, Adana, Turkey

Introduction: Insulin resistance, refers to decreased response of tissues to insulin, primarily associated with type 2 diabetes and glucose intolerance. Several results were obtained in previous studies about the relation between insulin resistance and thyroid dysfunction. In these studies hepatosteatosis was not an exclusion criteria despite insulin resistance has a key role in the development of it. In this study we aimed to investigate insulin resistance in patients with hypothyroidism or hyperthyroidism without hepatosteatosis.

Design: A total of 340 patients without hepatosteatosis were included. These patients were further divided into two study subgroups and a control group: 106 subjects with hypothyroidism, 104 with hyperthyroidism and 130 with normal thyroid function in the control group. The institution review board of hospital approved the study. We measured serum TSH, free T4, free T3 concentrations, blood glucose and insulin levels, serum lipid levels, hepatic transaminases and homeostasis model assessment of insulin resistance (HOMA IR). Insulin resistance was calculated according to HOMA index and compared between the groups. SPSS 19.0 package program (SPSS Inc., Chicago, Illinois) was used for statistical analysis. Ki square and ANOVA tests were used for comparing groups.

Results: Average age of 340 patients was $41.2 \pm 10.9$ years. Male/female ratio was 52:288. Frequencies of insulin resistance in hypothyroidism, hyperthyroidism or normal thyroid function were $17 \%, 20 \%$ and $24 \%(P=0.673)$, respectively. HOMA-IR indexes were $1.85 \pm 1.18,1.96 \pm 1.54,2.09 \pm 1.79$ respectively. The difference was not statistically significant $(P=0.589)$ (Table 1).

Conclusion: In this study, we did not found insulin resistance in patients with hypothyroidism or hyperthyroidism. We concluded that insulin resistance could be associated with comorbidities in patients with thyroid dysfunction in previous studies. In our opinion, hepatosteatosis should be evaluated as a comorbidity in such patients.

Keywords: Insulin resistance, hypothyroidism, hyperthyroidism, hepatosteatosis

\begin{tabular}{|lccccc|}
\hline Parameter & $\begin{array}{c}\text { Normal } \\
\text { Range }\end{array}$ & $\begin{array}{c}\text { Control } \\
(\mathrm{N}=130)\end{array}$ & $\begin{array}{c}\text { Hypothyroidism } \\
(\mathrm{N}=106)\end{array}$ & $\begin{array}{c}\text { Hyperthyroidism } \\
(\mathrm{N}=104)\end{array}$ & $P$ \\
\hline TSH (mcIU/ml) & $0,57-5,56$ & $1,83 \pm 0,98$ & $17,55 \pm 5,88$ & $0,08 \pm 0,08$ & 0,001 \\
$\begin{array}{l}\text { Insulin } \\
\text { resistance } \\
\text { frequency }\end{array}$ & - & $31(24 \%)$ & $18(17 \%)$ & $21(20 \%)$ & 0,673 \\
HOMA-IR & $<2,5$ & $2,09 \pm 1,79$ & $1,85 \pm 1,18$ & $1,96 \pm 1,54$ & 0,589 \\
\hline
\end{tabular}

Table 1: Comparison of groups according to the HOMA-IR and insulin resistance frequency. 